SHORT REPORT

\title{
Interlocking finger test: a bedside screen for parietal lobe dysfunction
}

\author{
L R Moo, S D Slotnick, M A Tesoro, D S Zee, J Hart
}

J Neurol Neurosurg Psychiatry 2003;74:530-532

This study sought to determine the utility of an interlocking finger task in screening for parietal lobe dysfunction. The ability of 69 patients to imitate a standardised set of four interlocking finger figures was compared with concurrent performance on formal neurocognitive tests. Poor interlocking finger test scores correlated most highly with standard measures of parietal lobe dysfunction. In addition, an analytical model of parietal dysfunction indicated the interlocking finger test was similar to, if not better than, standard tests of parietal lobe dysfunction. Attempts to imitate these figures should serve as a fast and simple screen of parietal lobe dysfunction.

\footnotetext{
$\mathrm{F}$
} ormal neurocognitive test batteries provide invaluable information regarding specific cognitive domains, allow for comparison to established norms, and reveal patterns of deficits consistent with a variety of neurological diagnoses. Their use is often limited because they require a trained person and lengthy time to administer. Simple screening tests that can be incorporated into the bedside or office based neurological examination are useful if they ascertain whether or not more detailed investigations are indicated.

While a general impression regarding level of consciousness, orientation, and language can often be made while taking a history, casual assessment of functions predominantly subserved by the parietal lobes is more difficult. A variety of formal cognitive tests can assess parietal lobe function, and typically include visual-constructional tasks (for example, Rey-Osterrieth Complex Figure, drawing interlocking polygons (as on the MMSE), clock drawing/setting), visual perceptual tasks (for example, time perception, Benton Judgement of Line orientation, Ravens Progressive Matrices, Hooper Visual Organization test), right-left orientation, and arithmetic/calculations (WAIS-R performance subtest). ${ }^{1}$ However, most of these tests require considerable time and expertise to administer in addition to equipment that may not be available in a clinic or emergency department setting.

Imitation of finger and hand figures has been used to test visual-spatial skills, ${ }^{2-5}$ although without clear anatomical localisation or correlations to other standardised tests. Non-symbolic gesture imitation is frequently used as it has been shown to be impaired more frequently than imitation of learned gestures or those with symbolic meaning (for example, saluting, hitchhiking). ${ }^{4}$ Imitation of finger configurations specifically, in contrast with whole hand gestures, has been found to be impaired with equal frequency in both left and right brain damaged patients, ${ }^{6}$ and thus seems to have little cerebral lateralisation.

We hypothesised that a task that entails imitation of a series of finger gestures without symbolic meaning would require visual-spatial integration and correlate highly with other measures of parietal lobe function for the following reasons: (1) single cell recording results in non-human primates indi-

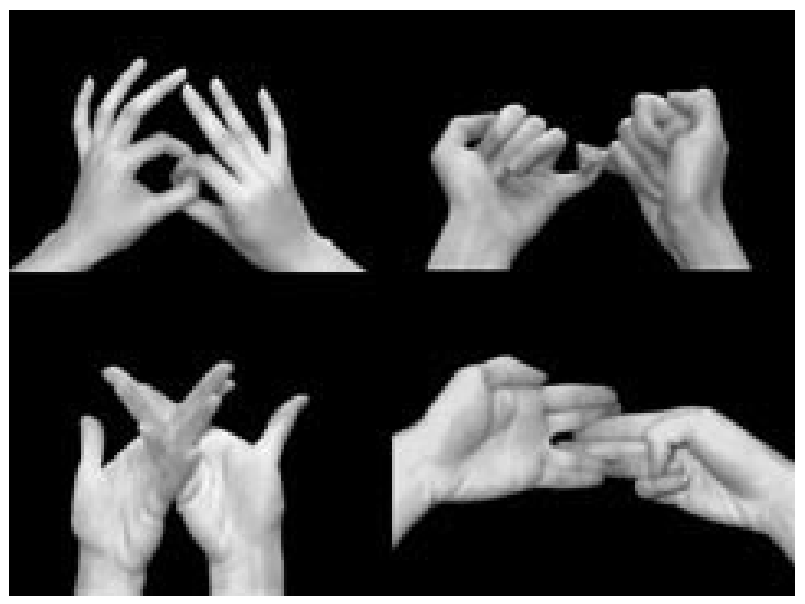

Figure 1 Examiner's hands demonstrating the four interlocking finger figures.

cate that posterior parietal lobe structures are responsible for the integration of visual information, upper extremity movement and proprioception, ${ }^{7}(2)$ there is evidence that focal lesions in posterior parietal cortex result in lack of coordinated movements of the hands under visual guidance (optic ataxia), ${ }^{8}$ and (3) functional imaging of normal participants during imitation of similar non-symbolic finger configurations demonstrates bilateral supramarginal gyrus activation (within the parietal lobe). ${ }^{9}$ To test our hypothesis regarding the link between finger imitation and parietal dysfunction, we standardised the interlocking finger figures and correlated them with various cognitive tests from our clinical battery.

\section{PARTICIPANTS AND METHODS}

Participants included the 69 consecutive patients ( 33 female, mean age 70 years with range 43-92), without upper extremity paresis, who underwent both neurological examination and neuropsychological testing in the Johns Hopkins Outpatient Cognitive Neurology Clinic between 1998 to 1999. The diagnoses of this patient population included dementias, focal cortical degeneration, epilepsy, herpes simplex encephalitis, post-radiation therapy complications, stroke, and pseudodementia of depression. As part of the mental status portion of the neurological examination, patients were instructed to imitate each of the four interlocking finger figures, one at a time, as the examiner demonstrated the figures (see fig l).

The four figures were uncommon and without symbolic meaning. The examiner directly faced the patient during presentation, and continued to demonstrate each figure until the patient felt that they had accurately reproduced it. The ability to imitate the four figures was scored with one point for each correctly reproduced figure, with total scores being whole numbers ranging from 0 to 4 . Figures were considered correct 
Table 1 Spearman correlation of interlocking finger figure scores with other test scores

\begin{tabular}{lll}
\hline Test/clinical feature & $\mathrm{n}$ & $r_{\mathrm{s}}^{2}$ \\
\hline Clock setting & 30 & $0.2313^{* *}$ \\
Clock face construction & 31 & $0.2141^{* *}$ \\
Time perception & 25 & $0.2366^{*}$ \\
Rey-Osterrieth Complex Figure copying & 31 & $0.1854^{*}$ \\
MMSE total & 38 & $0.1174^{*}$ \\
Arithmetic (WAIS-R subtest) & 23 & 0.1617 \\
Trails Making Test part B centile & 17 & 0.1432 \\
Boston Naming Test & 35 & 0.1040 \\
Grooved pegboard centile (dominant hand) & 25 & 0.0609 \\
Sex & 38 & 0.0538 \\
Verbal fluency centile (FAS) & 33 & 0.0318 \\
Digit span (age corrected scaled score) & 36 & 0.0253 \\
Grooved pegboard entile (non-dominant hand) & 23 & 0.0120 \\
Age & 38 & 0.0054 \\
RAVLT 5th trial & 33 & 0.0005 \\
\hline
\end{tabular}

For each neurocognitive test/demographic feature, the number of subjects included in the analysis ( $n$ ) and the square of the Spearman correlation $\left(r^{2}\right)$ of the interlocking finger figure scores with the given test score is reported. Tests were ranked from top to bottom initially in decreasing order of significance ( $p$ value) and then by decreasing correlation of scores within each significance level. ${ }^{*}$ *Significant at $p<0.01$. * Significant at $\mathrm{p}<0.05$

when the interlocking finger component was accurate regardless of positioning of the non-interlocking fingers or posture of the arms.

After taking the patient's history and an examination, patients spent the next two to three hours undergoing an extensive battery of formal neurocognitive tests, administered and scored by a technician (see table 1). These generally included tests of global function (the MMSE, attention/ concentration, psychomotor speed), frontal function (executive function), fronto-temporal function (memory and language), and parietal lobe function (arithmetic, visual-spatial/ visual-constructional domains). For example, within the visual-spatial domain, patients were instructed to draw an analogue clock face from memory, placing all of the numbers in their proper positions ("clock drawing"). This was scored on a scale of $1-10$ with points subtracted for a poorly formed circle, numbers placed in the incorrect quadrant, missing numbers, etc. In addition, patients were instructed to set the clock ("clock setting") by drawing in the hands such that the time read "ten after eleven". Time perception was assessed by having patients read a series of photographs of analogue clock faces.

Two methods were used to assess the degree of correspondence between the interlocking finger figure scores and other tests of neuropsychological dysfunction: (1) a simple correlation and (2) similarity to other parietal lobe tests at predicting parietal dysfunction in an analytical model. In the first method of assessment, total interlocking finger figure scores were correlated with each domain specific standard neurocognitive test score. As the goal was to obtain a measure of consistency of relation, without assuming a linear relation, a Spearman correlation was calculated. A two tailed test of $r_{\mathrm{s}}^{2}$ was conducted to test for a significant correlation at the $\mathrm{p}<0.05$ and the $\mathrm{p}<0.01$ levels. The correlation between scores of patients with both perfect interlocking finger-figure scores (4/4) and high neurocognitive test scores often resulted in artefactually significant correlations; therefore, patients with perfect interlocking finger figure score were excluded from the analysis. This resulted in an $\mathrm{n}<69$ for each correlation between interlocking-finger-figure scores and the scores for the neurocognitive test of interest.

In the second method of assessment, on a patient by patient basis, the performance on each standard parietal lobe test (that is, clock setting, clock face construction, time perception, Rey-Osterrieth complex figure copying, arithmetic), was labelled as impaired or unimpaired using a conservative criterion (for example, a score $<2$ standard deviations below the mean). Each patient was defined as having parietal lobe dysfunction if they demonstrated impairment in at least two parietal lobe tests. In this way, parietal lobe dysfunction was modelled analytically, thus allowing for statistical assessment of the ability of any one test to predict parietal lobe dysfunction (as indicated by the other tests). To assess the utility of the interlocking finger figure test, for example, impaired performance on at least two of the performed standard tests of parietal function was used and the corresponding sensitivity, specificity, positive predictive value (PPV), and negative predictive value (NPV) of the interlocking finger test was calculated. A similar assessment was made for each conventional parietal lobe test using the other standard parietal lobe tests to model dysfunction. These five sets of statistical results were used to quantify conventional parietal lobe test variability to be used for comparison with the interlocking finger figure scores.

\section{RESULTS}

The four neurocognitive tests that correlated best with performance on the interlocking finger figure task were all primarily tests of parietal lobe function (that is, the specificity and PPV of the interlocking finger figure task for parietal lobe dysfunction was high). (See table 1.) Clock setting and clock face construction were the most highly correlated $(p<0.01)$, followed next by time perception, Rey-Osterrieth complex figure copying, and the MMSE score $(\mathrm{p}<0.05)$.

As would be expected, no correlation between interlocking finger performance and age or sex was found. There was also no significant correlation between interlocking finger figure performance and tests believed to primarily assess nonparietal lobe functions (for example, language, memory, attention, or psychomotor speed).

Using the analytical model, interlocking finger figure sensitivity was equal to 0.88 , specificity was equal to 0.64 , PPV was equal to 0.58 , and NPV was equal to 0.90 . These values were similar to or better than all of the standard tests of parietal lobe dysfunction (with similarity defined as being within one standard error of the mean). While interlocking finger figure specificity was within one standard error of the specificities of the standard parietal lobe tests, interlocking finger figure sensitivity, PPV, and NPV were all at least one standard error above the conventional parietal lobe test results.

\section{DISCUSSION}

Poor performance on the interlocking finger figure task was most correlated with impairment on parietal lobe tests during 
formal neurocognitive testing. Every neuropsychological test that was significantly correlated with the interlocking finger figure task is considered primarily a parietal lobe task-clock face construction, clock setting, time perception, and ReyOsterrieth complex figure copying. Arithmetic, while highly correlated with the interlocking finger task did not reach significance presumably because of a lack of power (see table 1). Imitation of finger figures probably entails a combination of limb praxis, optic praxis, visual-spatial, and visuoconstructional skills. Each of these domains has been linked to the parietal lobe (for a review see Benton ${ }^{10}$ ). Thus, the correlation between the task and various parietal lobe tests is consistent with previously described neuroanatomical and neurobehavioural relations.

Difficulty with comparable hand or finger tasks has also been described in patients with frontal lesions, but detailed analysis has demonstrated this was true only when rapid sequencing through multiple hand positions was required. ${ }^{5}$ Frontal lobe contributions to finger and hand movement have been proposed to relate to gross execution of the motor task with the parietal lobe defining the precise details of the gestures. ${ }^{11}$ Lack of significant correlation with neuropsychological tests measuring primarily frontal lobe function in this study, such as verbal fluency or Trails B, also argues against the interlocking finger figure task being principally a measure of frontal lobe dysfunction. Despite our task being minimally dependent on language, memory, or attention, there was clearly some relation to global measures/performance such as the MMSE (see table 1). Covariance of the MMSE total score and other tests is usually indicative of a correlation between global decline and the tasks under investigation, which was probably the case here.

Clearly no single neurological or neuropsychological test in isolation can precisely localise a disorder and conversely, no cognitive function relies exclusively on a single cognitive domain. Even here, although the interlocking finger test was better in most regards than conventional parietal lobe tests, its specificity was not high (even though it faired at least as well as conventional parietal lobe tests). Therefore, a less than perfect score on the interlocking finger test should be further defined with formal cognitive testing. As with all clinical assessments, multiple complementary tests need to be administered and considered together to precisely assess neuropsychological dysfunction. That said, the interlocking finger figure test not only correlates highly with other established tests of parietal lobe dysfunction, but equals or surpasses the capacity of any other single test to assess such dysfunction. Given this, and the ease and speed of its administration, it should serve as a useful initial bedside screen for parietal lobe dysfunction.

\section{Authors' affiliations}

L R Moo, M A Tesoro, D S Zee, Department of Neurology, Johns Hopkins School of Medicine, USA

S D Slotnick, Department of Psychology, Harvard University, USA

J Hart, Departments of Geriatrics, Neurology, and Radiology, University

of Arkansas Medical School, USA

Competing interests: none declared.

Correspondence to: $\operatorname{Dr} L \mathrm{R} M \mathrm{Mo}^{\dagger}$, Department of Neurology, Johns Hopkins University, 600 N Wolfe Street, Meyer 100, Baltimore, MD, USA; Imoo@jhmi.edu

${ }^{\dagger}$ Currently visiting scholar at Harvard University: c/o Harvard University, 33 Kirkland Street, Room 918, William James Hall, Cambridge MA 02138, USA

Received 29 August 2002

Accepted in revised form 15 November 2002

\section{REFERENCES}

1 Lezak MD. Neuropsychological assessment. 3rd edn. NewYork: Oxford University Press, 1985.

2 Damasio AR, Benton AL. Impairment of hand movement under visual guidance. Neurology 1979;29:170-8.

3 De Renzi E, Motti F, Nichelli P. Imitating gestures: A quantitative approach to ideomotor apraxia. Arch Neurol 1980;37:6-10.

4 Dee HL, Benton AL, Van Allen M. Apraxia in relation to hemispheric locus of lesion and aphasia. Transactions of the American Neurological Association 1970;95:147-50.

5 Jason GW. Performance of manual copying tasks after focal cortical lesions. Neuropsychologia 1986;24:181-91.

6 Goldenberg G. Defective imitation of gestures in patients with damage in the left or right hemispheres. J Neurol Neurosurg Psychiatry, 1996:61:176-80.

7 Eskandar EN, Assad JA. Dissociation of visual, motor and predictive signals in parietal cortex during visual guidance. Nature Neuroscience 1999;2:88-93.

8 Yin TC, Mountcastle VB. Visual input to the visuomotor mechanisms of the monkey's parietal lobe. Science 1977;197:1381-3.

9 Tanaka S, Inui T, Iwaki S, et al. Neural substrates involved in imitating finger configurations: an fMRI study. Neuroreport 2001;12:1171-4.

10 Benton A. Visuoperceptive, visuospatial, and visuoconstructive disorders. In: Heilman KM, Valenstein E, eds. Clinical neuropsychology. NewYork: Oxford University Press, 1979:186-232.

11 lacoboni M, Woods RP, Brass M, et al. Cortical mechanisms of human imitation. Science 1999:286:2526-8. 